\title{
Preface: special issue on computational micromechanics of materials
}

\author{
Javier Segurado • Tomasz Sadowski • \\ Javier LLorca $\cdot$ Siegfried Schmauder
}

Published online: 26 October 2015

(C) The Author(s) 2015. This article is published with open access at Springerlink.com

Engineering structural materials, from metallic alloys to composites and to polymers are not homogeneous and present complex microstructures. Their mechanical behavior is closely linked to their particular microstructure and its evolution with loading.

A detailed understanding and prediction of the macroscopic mechanical behavior implies the use of mathematical models that account for the links between the microstructure and the deformation

J. Segurado · J. LLorca

IMDEA Materials Institute, C/ Eric Kandel 2,

28906 Getafe, Madrid, Spain

e-mail: javier.segurado@imdea.org

J. LLorca

e-mail: javier.1lorca@imdea.org

J. Segurado · J. LLorca

Department of Materials Science, Polytechnic University

of Madrid, E. T. S. de Ingenieros de Caminos,

28040 Madrid, Spain

T. Sadowski $(\bowtie)$

Faculty of Civil Engineering and Architecture, Lublin

University of Technology, Nadbystrzycka 40,

20-618 Lublin, Poland

e-mail: t.sadowski@pollub.pl

\section{S. Schmauder}

Institute of Materials Testing, Materials Science and

Strength of Materials, University of Stuttgart,

Pfaffenwaldring 32, 70569 Stuttgart, Germany

e-mail: siegfried.schmauder@uni-stuttgart.de mechanisms that operate at different length scales. Moreover, the macroscopic behavior usually involves mechanisms that take place at very different length and time scales and the models should be able to couple these mechanisms. The models that connect the microstructure with the mechanical behavior are in general complex and the resulting equations have to be solved using computational tools.

In the last decades, in parallel with the increase in computational power, Computational Mechanics of Materials has become one of the most active areas in Material Science research. In particular, Micromechanical models that analyze the mechanical response at the $\mu \mathrm{m}$ scale to link the microstructure with macroscopic mechanical behavior are well established.

The International Workshop Series on Computational Mechanics of Materials has pioneered the activities in Computational Mechanics with special attention to the research based on micromechanical approaches. Its first edition was celebrated in 1990 and the workshop has been organized yearly since then. Nowadays, it has become a classic forum in the computational mechanics community to discuss the latest advances and the future research directions in various areas pertaining to computational mechanics of materials. These workshops are intended to cover all aspects of modeling and simulation of the mechanical behavior at different length and time scales. The 
materials of interest range from traditional structural materials (such as metals, alloys, polymers and composites) to novel materials and bio-materials. In October 2014, the workshop was organized in Madrid by IMDEA Materials Institute and over 70 talks were presented by researchers from all over the world.

This special issue on "Computational Micromechanics of Materials" contains a selection of some of the talks presented in the workshop. The articles in this issue cover different length scales ranging from atomistics to macroscopic structural mechanics and also include several papers dealing with multiscale approaches. The materials under study are also diverse including metals, composites, concrete and polymers.

We hope that the papers in this issue will provide opportunities to identify and discuss future developments in the field of Computational Micromechanics of Materials. Special thanks are directed to the former and present editors of Meccanica, Prof. Alberto Carpintieri and Prof. Luigi Gambarotta for giving us the opportunity of to act as guest editors of this special issue of Meccanica.

Open Access This article is distributed under the terms of the Creative Commons Attribution 4.0 International License (http:// creativecommons.org/licenses/by/4.0/), which permits unrestricted use, distribution, and reproduction in any medium, provided you give appropriate credit to the original author(s) and the source, provide a link to the Creative Commons license, and indicate if changes were made. 